\title{
La práctica educativa del Trabajo Social en un contexto de encierro
}

Miguel Ángel Oliva Carreras

Lic. en Trabajo Social (UNCa)

E-mail: miguelangeloliva@gmail.com 
Resumen

La enseñanza en un contexto de encierro como es el Servicio Penitenciario, se desarrolla en el marco de una capacitación, acompañamiento y asistencia técnica participativa, con un sentido solidario, considerando que desarrollamos la tarea en un escenario complejo, en el cual los internos reciben la educación universitaria dentro de la institución. Procurando establecer un puente que les facilite y sirva de nexo con la sociedad de tal manera de fortalecer su espíritu, valorar su decisión de estudiar y sentir que es un paso hacia su libertad. Promoviendo un trabajo grupal, el cual permite interactuar entre ellos y con nosotros, aprendiendo por nuestra parte el sentido profundo de la experiencia en la cual tenemos la oportunidad de participar, en un "enseñar a enseñar" para "aprender a aprender". La capacitación se da desde una perspectiva eminentemente dialógica y participativa, con y desde los internos involucrados, la evaluación es permanente desde el inicio a fin de disponer de un diagnostico participativo inicial, que nos permita conocer la situación -nivel de instrucción- del colectivo y cada sujeto en particular. Siendo el objetivo general desarrollar una actitud reflexiva y creativa que posibilite la resolución de los problemas y la transformación de la realidad.

\section{Palabras claves}

Trabajo Social - práctica educativa - contexto de encierro
Abstract

Education, in a context of closure, prison service, takes place in the framework of a training, accompaniment and support participatory, with a sense of solidarity, considering that we develop the task in a complex scenario, where inmates receive college education within the institution. Seeking to establish a bridge that facilitates them and serve as a link with society. So strengthen your spirit, valuing his decision to study and feel that it is a step towards their freedom. Promoting a group work, which allows you to interact with each other and with us, learning our profound sense of experience in which we have the opportunity to participate, in a "teaching to teach" to "learning to learn". Training is given from a perspective essentially Dialogic and participatory, with and from the involved inmates, evaluation is permanent from the beginning to provide of a diagnostic participatory initial, that will allow us to know the situation - level of education - collective and each subject in particular. Being the general objective, to develop a reflective and creative attitude that facilitates the resolution of problems and the transformation of reality.

Key Words

Social work - educational practice - context of closure 
El presente trabajo se propone realizar una breve descripción de las posibilidades y limitaciones que se le presentan al trabajador social en la práctica educativa en un contexto de encierro, el cual es un escenario complejo, terreno concreto que nos permite construir y explicitar el ver, la mirada del Trabajador Social, cuando aborda y trabaja en el mismo, considerando las diferentes implicancias que se producen como resultado del impacto del contexto sobre cada uno de los actores y, por qué no decirlo, sobre el profesional actuante.

Surge a partir de la implementación de un programa denominado "La Facultad de Humanidades en la Cárcel” que se propone generar y establecer vínculos institucionales entre la Facultad de Humanidades y el Servicio Penitenciario Provincial, que coadyuven a la construcción de capacidades y pasividades de reinserción social de los internos.

\section{Políticas para los contextos de privación de la libertad}

"Esta modalidad del sistema educativo centra su tarea en garantizar el derecho a la educación de todas las personas privadas de libertad para promover su formación integral y desarrollo pleno. Incluye a quienes están involucrados en procesos judiciales, en carácter de procesados o condenados, y se encuentran alojados en instituciones de encierro. El ejercicio del derecho a la educación no admite ningún tipo de limitación ni discriminación relacionada con la situación de privación de la libertad. El acceso al sistema educativo y a la vida cultural en condiciones dignas, contribuyen a la inclusión social".

En el ámbito de un contexto de encierro, nos hemos insertado con el fin de introducir, en este caso en particular, a los alumnos interesados en iniciarse en la carrera de Licenciatura en Trabajo Social a través de una propuesta pedagógica como es el Modulo de Articulación Común, que es el mismo que se dicta a los alumnos que se inician

1 Programa La Facultad de Humanidades en la Cárcel s/expte F.H. N’362/10. El Programa es coordinado por el Mgter. Mario Alanis y aprobado por Resolución C.D.F.H.N' 050/05. 
en el ámbito de la Universidad Nacional de Catamarca, Facultad de Humanidades, Departamento de Trabajo Social.

Nos interesa a continuación exponer algunas consideraciones comunes de una clase participativa. El aula, el dictado de una clase, es lo que nos permite tener un primer contacto con los alumnos, presentarnos e iniciar la actividad a través de un módulo introductorio. Se pretende de esta manera lograr una comprensión práctica y teórica del significado del Trabajo Social, su articulación, implementación y aplicación en el terreno concreto.

Es una manera de sustituir el "trabajo de campo" haciendo "ver" la realidad que se explicita en los distintos escenarios, insertos en los espacios poblacionales, heterogéneos, complejos, dinámicos en los cuales se desenvuelve la comunidad organizada.

Con la participación de los alumnos, se analizaron los textos que constituyen el módulo introductorio, a fin de visualizar la comprensión de estos parte de los mismos, la explicación de lo comprendido, el significado que los autores dan a sus expresiones y su vinculación con la realidad que en cada oportunidad se debe abordar, así como la identificación de las 'situaciones-problemas' que deben ser problematizadas. Se incentivó el trabajo grupal en el aula y el trabajo individual por razones prácticas, considerando el tiempo-espacio que cada alumno dispone en su ámbito.

El Trabajo Social reconoce la fuerza del grupo e intenta canalizarla hacia una acción constructiva. El grupo se convierte en el instrumento gracias al cual se puede conseguir dos objetivos: ayudar a los individuos a utilizar el grupo para las necesidades de su propio desarrollo y ayudar al grupo para realizar una acción socialmente deseable (ONU, 1960:12).

En 1949 el comité de la Asociación Norteamericana de Trabajadores Sociales publicó un informe donde se definían sus funciones, objetivos y técnicas. El Trabajo Social de grupo centró su interés en tres aspectos fundamentales: en los objetivos de participación social y educativa; en la socialización y el desarrollo social para la realización individual y para preparar a la persona a asumir roles sociales responsables; y en el tratamiento o rehabilitación del pequeño 
grupo como medio de modificar la propia conducta y llegar a la realización personal. Se incrementa la literatura y aparecen aportaciones importantes como las de Konopka, Trecker y Vinter.

En 1955 Las Naciones Unidas publica un folleto "El Progreso Social por el Desarrollo Comunitario" y organiza el primer seminario europeo sobre Trabajo Social de Grupos en Finlandia (Domenech, 1998:38). A partir de los años '70 se rompe con la estructura de especialidad con la integración de los tres métodos (caso, grupo, comunidad) y aparece una visión más global de la práctica profesional, fundamentalmente por la incorporación de la teoría de sistema y el análisis aportado por la sociología critica. Se percibe un acercamiento entre los continentes y se acaba con la hegemonía de este saber que procedía de América del Norte. Aparece la primera revista de Trabajo Social con Grupos publicada en 1988 en Inglaterra (Group Work) y se celebra el primer symposium Europeo en 1999.

En la década de los noventa el Trabajo Social con Grupos ha sufrido una nueva vitalización como producto de las nuevas necesidades que generan las sociedades complejas. "Nos encontramos inmersos en una sociedad caracterizada por la innovación tecnológica y por la transformación y adaptación de las formas de interacción social a un nuevo contexto. Se exigen mayores habilidades sociales, competencias técnicas y capacidad de adaptación, a la vez que aumenta la inestabilidad familiar y laboral. Y todo ello en un contexto caracterizado por un proceso de individualización en el que se debilitan los lazos sociales. En este medio histórico, el Trabajo Social con Grupos tiene una vigencia cada vez mayor, y permite recuperar o fortalecer nuestras capacidades de interacción social” (Fernández y López, 2006:24).

Frente a las situaciones de transformación y evolución de los problemas sociales (tecnológicos, demográficos, nuevos modelos familiares y lo que se ha dado en llamar la "nueva cuestión social" ligada al desempleo y la exclusión social), el Trabajo Social responde a través de las "nuevas políticas sociales, la redefinición de las funciones del Trabajo Social y una manera distinta de abordar ciertos campos de intervención” (De Robertis, 2003:171). 
La renovación metodológica, para Cristina de Robertis, viene marcada en primer lugar por la función a cumplir por el Trabajo Social en la actualidad. Una función de restablecer y crear el vínculo social, lo que significa la creación de oportunidades de intercambio, la dinamización de las redes primarias de las personas ayudadas, su participación en las actividades y en los lugares de vida social a nivel de vecindario, del barrio, o de la ciudad, y otra función de restaurar la ciudadanía y de empoderamiento (empowerment), para facilitar el acceso a los derechos sociales y cívicos así como un mayor poder a las personas beneficiarias de la intervención. Luego, por la nueva articulación de las intervenciones individual/colectiva para evitar la dicotomía entre lo individual y lo colectivo, que ha dado lugar a una renovación del Trabajo Social con Grupos y la intervención en redes. Por último, propone trabajar con los recursos y las fuerzas de la persona (De Robertis, 2003).

La definición de Trabajo Social con Grupos desarrollada por Konopka lo ubica como "un método del trabajo social que ayuda a los individuos a mejorar su funcionamiento social a través de experiencias de grupo planeadas y a hacer frente, de forma efectiva, a sus problemas personales, de grupos o comunidad" (Konopka, 1973:42). El énfasis está, sin embargo, en ser de ayuda para el individuo con problemas. "El Trabajo Social contemporáneo pone el acento en la acción y la influencia, como también en la interacción y adaptación. De alguna forma, la definición queda más completa si se añade lo siguiente: el Trabajo Social con Grupos proporciona un contexto en el cual los individuos se ayudan mutuamente; es un método para ayudar tanto a grupos como a individuos, y para conseguir que tanto individuos como grupos sean capaces de solucionar problemas personales, de grupos, de organización, y de la comunidad" (Vizcarret, 2007:45).

Existe cierta dificultad a la hora de marcar sus límites; Konopka y otros autores utilizan el término Trabajo Social con Grupos para describir el trabajo con grupos que pone el acento en el funcionamiento social y distinguirlo de la psicoterapia de grupo que pone el énfasis en las necesidades emocionales y en los procesos psicológicos. 
Otro problema de limitación se plantea con la terapia familiar (trabaja con la familia como un grupo) porque la familia es un tipo de grupo arquetípico y "natural" que tiene mucho en común con otros tipos de grupos del trabajo social, pero también muchas diferencias. Igualmente es difícil fijar el límite entre el Trabajo Social con grupos y el comunitario. El trabajo social con grupos de la comunidad se puede considerar un tipo de Trabajo Social de grupo cuando los grupos son pequeños y los objetivos son locales, y cuando el método da especial relevancia a los procesos e interacciones que se produce dentro del grupo (Vizcarret, 2007:45).

El Trabajo Social con Grupos utiliza la fuerza del grupo para fomentar la ayuda mutua y el desarrollo personal, y al grupo como micro-organización para promover la acción colectiva y la acción social. En términos generales, los objetivos de Trabajo Social con Grupos son básicamente evitar el aislamiento, fomentar el aprendizaje y la madurez social, preparar a los miembros para las crisis o cambios vitales, resolver o aclarar problemas a nivel personal/ familiar, resolver problemas del entorno del grupo, e influir a nivel social.

Cristina De Robertis en su análisis de diferentes prácticas de grupos, plantea dos modelos distintos de intervención grupal: uno, el grupo de desarrollo personal, que nos presenta el grupo como un lugar de "transición" para personas atomizadas que les permiten convertirse en integradas, "las personas pueden comunicarse con sus iguales en un clima de confianza mutua y de convivencia. La situación de grupo favorece el desarrollo personal de los miembros y la aparición de procesos de ayuda mutua" y otro, el grupo de desarrollo local, para afrontar los problemas colectivos, "las personas participan en calidad de habitantes de la localidad, sus otros status peyorativos o estigmatizantes pasan a un segundo plano. El grupo se convierte poco a poco en una estructura organizada y autónoma del profesional, que se integra socialmente y participa del vínculo comunitario" (De Robertis, 2003:177). Los dos modelos los podemos diferenciar en base a su énfasis sobre el proceso (el efecto que la participación en el grupo por sí mismo tiene sobre los miembros) o sobre las metas externas al grupo, con alguna forma de desarrollo y cambio social. 
Se produjeron debates interesantes, sobre distintos temas con vinculación directa o indirecta con el Trabajo Social. Se trabaja con insistencia en procurar el recupero de capacidades y saberes de cada uno de los alumnos, así como de la visión y misión del Trabajador Social y su relación con los distintos actores de la comunidad organizada.

Como parte de las primeras acciones que entendemos son necesarias a fin de evaluar la capacidad de los alumnos, se produce una evaluación diagnostica, mediante una serie de preguntas, simples, que el alumno, de manera breve y escrita debe contestar, lo cual nos permite tener una primera impresión de la capacidad de respuesta, con sus propias palabras y conocimiento, sin contar con elementos de ayuda, como un diccionario, por ejemplo.

Se dicta o entrega un texto, el cual debe ser leído y explicado, para producir una evaluación sumativa, que nos permita saber del grado de comprensión por parte de los alumnos participantes. Se produce una evaluación formativa, que se va rediseñando en cada oportunidad según las características del grupo y de cada uno de los alumnos.

La evaluación final se da como resultado de una evaluación permanente, que se logra gracias a la activa participación de los alumnos, pudiendo establecerse distinciones, lo cual se supera debido a que el Grupo superó la media y mostró sus capacidades con las consiguientes diferencias que cada uno presenta por razones de formación, dedicación y participación. Se evaluó conocimiento, comprensión, vocabulario especifico, formas de expresión oral y escrita, autonomía de juicio.

Entonces, el aula en tanto espacio de posibilidades, por medio del proceso de enseñanza-aprendizaje, constituye el inicio de un proceso de cambio, un espacio de libertad, dialogo, de interrelación participativa, de preguntas y respuestas, de evaluación, y diseñar un camino de características y perspectivas diferentes a las actuales, que le permitan a cada uno de los alumnos participantes imaginar una situación diferente.

En el tiempo y espacio disponible en el aula, se debe procurar visualizar, los detalles que explicitan el cuerpo, la mente y el espíritu de cada de uno de los estudiantes, que se introducen en lo que denomi- 
namos "espacio de libertad" e indudablemente lo hacen con la carga del contexto de encierro que impacta en los mismos, y que uno debe advertir a fin de direccionar la situación-problema de tal manera que no incida en el desarrollo de la actividad áulica

Cabe considerar y tener una opinión sobre el "aburrimiento", el cual implica otros factores que influyen en la distracción, la no participación, la ausencia, la deserción, la falta de contención. El error es creer que el alumno ingresa formado y en condiciones de integrarse y absorber el cambio que implica insertarse en un espacio de estudio, que lo recibe, lo cual implica que uno debe preocuparse por su desenvolvimiento, su desempeño, ya que si lo consideramos que está en condiciones de desenvolverse en este espacio diferente en el cual tomo la decisión de participar, nos equivocamos. Esto nos lleva necesariamente a la "motivación", lo cual implica cómo hacer para que los alumnos tengan interés por aprender aquello que se busca transmitir. Estar motivado es una condición propicia y deseable para el aprendizaje. Las tareas se hacen mejor cuando despiertan interés y desafíos. Las actividades son más ricas cuando los alumnos se involucran en ellas. El interés facilita la mayor concentración y el esfuerzo por lograr siempre más; su ausencia puede generar la indiferencia, la apatía, el abandono, y hasta el rechazo explicito

Lo que procuramos a través de este relato de experiencia es abrir una ventana hacia el interior del contexto de encierro e intentar que ustedes imaginen la secuencia de una clase en la cual están sentadas personas que explicitan de alguna manera su interés por la misma, uno tiene la oportunidad de tomar contacto con esa sensación, con esa necesidad colectiva, de que uno llegue e inicie la actividad, ya que la presencia de uno en ese ámbito les asegura la permanencia en el mismo, aunque sea por el efímero tiempo de dos horas, siendo tan emotiva la despedida como la recepción.

El alumno en este espacio tiene oportunidad de expresar lo que siente, y uno facilita que esa expresión se dé libremente, en un marco de respeto tácitamente acordado por todos los que participativamente estamos trabajando con un criterio de "todos enseñan - todos aprenden" lo cual no es necesario insistir, casi se podría decir ni corregir, 
salvo mantener con la mirada lo acordado de no excedernos en el uso de la libertad que nos da el espacio en el cual nos desenvolvemos, preservando de esa manera la continuidad de esa idea, que tiene el significado profundo de aportar un granito de arena a la tarea de sumar esfuerzos que implican comenzar a caminar un nuevo camino, a tener la capacidad de superar los inconvenientes y tropiezos que cada uno deberá con seguridad enfrentar para poder de esa manera alcanzar el objetivo propuesto

No tenemos ni expresamos prejuicios, consideramos que nos estamos desenvolviendo, de una manera similar a como lo hacemos en el ámbito de la Universidad, lo cual le permite a uno hacer la tarea, sin pensar en la complejidad de la situación en la cual nos desenvolvemos, si uno no tiene la capacidad de poder hacerlo de esta manera, entendemos, que transmitiríamos, miedos, o temores que serían rápidamente descubiertos y la relación, la comunicación se vería entorpecida, por esta actitud de nuestra parte.

Como afirma Alejandra Birgin, "creemos fuertemente que es posible romper con la inexorabilidad y tenemos la convicción de que las cosas pueden ser de otro modo" (2004:9). Si generamos las condiciones para cambiar las tendencias, los destinos no están necesariamente marcados de antemano. La cultura y la educación son las herramientas genuinas y obligadas para disminuir los efectos del encierro sobre los sujetos, restituir los derechos vulnerados incluso tempranamente, y abrir posibilidades para construir futuros diferentes a los asignados socialmente.

Como producir la iniciación de un proceso de cambio es el interrogante, deseamos comentarles cómo lo entendemos, cómo visualizamos la situación problema del colectivo, como individual, de cada uno de los que tomaron la decisión de involucrarse en la tarea de aprender a aprender. 


\section{Calidad como transformación}

Esta noción se basa en el "cambio cualitativo", cuestiona la idea de calidad centrada en el producto, pues considera que la calidad radica, por un lado, en desarrollar las capacidades del consumidor, estudiante, $y$, por otro, en posibilitarle para influir en su propia transformación. En el primer caso, el "valor agregado" es una medida de calidad, en términos del grado en que la experiencia educativa incremente el conocimiento, las capacidades y las destrezas de los estudiantes. En el segundo, supone una implicación del estudiante en la toma de decisiones que afectan su transformación que, a la vez, proporciona la oportunidad de ampliar sus posibilidades para participar en la vida profesional.

Esta idea de calidad como transformación cuestiona la relevancia del enfoque de calidad centrado en el producto a la educación superior (Elton, 1992), dado que la educación no es un servicio para un cliente, sino que un proceso continuo de transformación del participante, sea estudiante o investigador. Por ende, esto lleva a dos conceptos de calidad transformacional en educación: el enriquecimiento del consumidor y el reforzamiento del consumidor.

Una educación de calidad es aquella que efectúa cambios en el participante y presumiblemente lo enriquece. Esta noción de "valor agregado" otorga un sentido sumativo a este enriquecimiento (Astin, 1985), pues el "valor agregado" es una medida de cualidad en tanto la experiencia educacional enriquezca el conocimiento, las habilidades y destrezas de los estudiantes, (Goverment, 1991). Por lo tanto, una institución de alta calidad es aquella que en gran medida, enriquece a sus estudiantes (Astin, 1990), de modo que la determinación del valor agregado depende de la metodología y lo que se define como valor.

Cuando se mide el "valor agregado", por ejemplo en términos de la formación o conocimientos a la entrada y salida del proceso, se obtiene un indicador cuantificable de "valor agregado", pero se ignora la naturaleza de la transformación cualitativa.

El segundo elemento de la calidad transformativa es la entrega de poder que se da al alumno (Harvey y Burrows, 1992), lo cual implica 
otorgar poder a los estudiantes para influir en su propia transformación, se involucra al estudiante en el proceso de toma de decisiones que afecta su propia transformación. Tal como lo señalan Muller y Funnell (1992) "en cierta medida el que aprende debe apropiarse del proceso de aprendizaje y adquirir responsabilidad en la determinación del estilo y forma de entrega del aprendizaje”. Además, el proceso de transformación provee la oportunidad de autofortalecimiento con consecuencias positivas en el propio proceso de toma de decisiones (Roper, 1992).

El trabajo de Arthur Chickering (1978) sobre el impacto que tiene la educación universitaria en jóvenes adultos, avala también esta afirmación. Algunas maneras de otorgar poder a los estudiantes y hacerlos protagonistas de su propia transformación son: la evaluación de la docencia de los estudiantes, la selección de algunas actividades curriculares como el desarrollo de pensamiento creativo. Esto requiere que el estudiante sea tratado como actor intelectual y no como un simple receptor de información.

Por lo general, los alumnos asistentes no están en el mismo pabellón y el aula se convierte en un lugar de encuentro, intercambio, diálogo, conocimiento del uno y del otro, uno es partícipe de esas emociones, las cuales se manifiestan de la misma manera cuando llegamos o cuando nos retiramos, es una bienvenida, es una despedida, todo el tiempo nos acompaña la incertidumbre, estaremos todos en la próxima clase, nadie considera ese detalle, el imprevisto está latente en todo momento, pero no interfiere por lo menos de manera evidente en el tiempo áulico de que disponemos.

Trabajamos desde el Trabajo Social, colaborando en la construcción de una nueva historia de vida de cada uno, sin preguntar cuál es la que se debe dejar atrás, salvo que alguno tome la decisión de comentarla, o darnos detalles de la misma, respondemos a las preguntas o interrogantes que cada uno tiene, y tratamos de bajar los marcos teóricos a la realidad de la cotidianeidad que nosotros caminamos, que no es igual a la cotidianeidad del que sólo camina los espacios que le permite el contexto, que si bien es parte del contexto general, 
está aislado del mismo, solo es relatado y el otro toma conocimiento por lo que le dicen, pero no por sus propios medios.

El Trabajo Social es parte de la cotidianeidad de los espacios poblacionales heterogéneos, uno se desenvuelve en un trabajo de campo permanente, tomando contacto con la diversidad, e incidiendo a fin de visualizar lo que está presente y se manifiesta y no es visto por los decidores, esa realidad uno debe transmitirla, para que el espacio áulico sea el espacio de práctica, y en el mismo uno debe dibujar las diferentes situaciones problemas en las que uno tiene oportunidad de actuar o conocer, a fin de que cada uno imagine y explique cómo actuaría, cómo abordaría y cómo resolvería el tema en el cual debe actuar; si no se logra hacer esta tarea no hay una formación integral, que entendemos es necesaria y es compleja.

La educación superior universitaria en un contexto de encierro, debemos considerar que es un "proceso en construcción" y que se deben diseñar elementos que nos permitan evaluar ese proceso a fin de producir las correcciones que se estimen necesarias.

Se debe analizar el contexto y la coyuntura en que se plantea el problema de evaluar los resultados, uno de los problemas básicos para tal análisis radica precisamente en la definición de las dimensiones en la que habremos de estimar éxito/fracaso y lo que es aún más dificultoso, ya que implica una decisión axiomática, cual es el peso relativo que debe asignarse a cada una de esas dimensiones. En otras palabras: qué habremos de considerar un éxito o un fracaso.

Finalmente, plantear una intervención social con grupos requiere tener en cuenta los principios que orientan la intervención en Trabajo Social con Grupos, en este caso en un contexto de encierro. Los principios éticos que regulan la acción de los profesionales se derivan de las declaraciones y convenciones internacionales de Derechos Humanos. A partir de este marco convenido internacionalmente podemos plantear los dos grandes principios, que articulan la acción de los profesionales, establecidos en el código de Ética de la FITS: el Trabajo Social se basa en el respeto de los derechos humanos y la dignidad humana y promueve la justicia social, en relación con las personas y con la sociedad. 
Cristina de Robertis propone un concepto de persona amplio y complejo, que interesa especialmente al Trabajo Social con grupos, ya que pone el acento en superar la dimensión individual de la persona y presta atención a su dimensión social y colectiva: "Así la persona no es ya una individualidad única y diferente a todas las demás, sino que al mismo tiempo, es igual a todas las otras personas de su entorno social, tiene los mismos condicionantes socioeconómicos, los mismos derechos, las mismas obligaciones y, en cierta medida, aspiraciones semejantes. El acento se pone en lo común y no en lo que distingue" (2003:61).

La Asociación para el Avance del Trabajo Social con Grupo es una organización profesional internacional que busca promover y hacer progresar la práctica del Trabajo Social con Grupos. Esta organización ha formulado una serie de estándares generales para dicha práctica. El apartado primero identifica los siguientes conocimientos y valores fundamentales:

- Es importante conocer el contexto familiar, político y cultural de los miembros, así como su interacción, sus preocupaciones y, además, han de ser vistos como ciudadanos capaces de cambiar y de ayudar a los demás.

- La atención al individuo ha de ser desde una perspectiva sistémica, tanto en la intervención como en la evaluación. Para ello, hay que tener en cuenta la realidad bio-psico-social del integrante del grupo en su comunidad.

- La evaluación ha de estar basada en las competencias y ha de ponerse especial énfasis en los puntos fuertes de los miembros, así como han de conocerse sus puntos débiles.

- La función de ayuda mutua en el grupo está formada por múltiples relaciones de ayuda. El papel principal del coordinador es ayudar a los miembros a que se ayuden entre sí.

- Los grupos, caracterizados por procesos democráticos, ayudan a los miembros a asumir la responsabilidad del desarrollo del grupo. El coordinador y los miembros tienen el mismo peso; este, el coordinador, no es un "experto" todopoderoso dentro del grupo, por ello, ha de establecer relaciones recíprocas e igualitarias. 
- El énfasis en el empoderamiento. Los objetivos grupales realizan el crecimiento personal y el cambio social. El trabajador/a social promueve la autonomía tanto del grupo como de los individuos.

- El comportamiento de los grupos pequeños ha de ser analizado como una entidad separada y diferenciada de cada uno de los integrantes. Durante la vida del grupo se producen cambios que forman parte del proceso grupal que se dan en fases, todo esto influye en la conducta individual de los miembros.

- Los grupos formados, con distintos propósitos y objetivos (educación, resolución de problemas, acción social), influyen en lo que hace el coordinador de la misma manera que influyen en el grupo para la consecución de sus objetivos.

- El control y evaluación del éxito del grupo a la hora de conseguir los objetivos ha de hacerse mediante la observación y la medición de los resultados y/o procesos.

\section{Algunas ideas de cierre}

Debemos considerar que los intereses y las preocupaciones, no es fácil saber cuál es el pensamiento profundo de jóvenes o adultos que están permanentemente impactados por el contexto en el cual se encuentran. Lo que sí uno debe saber es que debe trabajar con el criterio de que "lo imposible es posible", no se admite la frustración.

No tenemos experiencias con jóvenes. El adulto responde a distintos intereses particulares, que lo motivan a participar en una actividad áulica, pudiendo visualizarse en algunos una motivación, un interés bastante acentuado en "aprender a aprender" a fin de superar sus propias contingencias y que le permitan iniciar un nuevo proyecto de vida. Las características, expectativas o necesidades particulares, deben ser visualizadas a través de un diagnóstico de evaluación inicial el cual nos va a indicar qué debemos tener en cuenta.

Entendemos que es muy importante producir una articulación participativa, en función de que la suma de opiniones nos va a permi- 
tir trabajar de una manera distinta en la implementación de criterios y tareas

El incentivo de la participación debe darse como resultado de una focalización a través de una mirada integral, que nos permita visualizar cuál/cuáles son los intereses de jóvenes y adultos, que den lugar a una sinergia contagiosa, de motivación y compromiso, si no hay compromiso con la tarea, posiblemente se va a producir la tarea, por razones especulativas y así no se logra construir una base sólida, un cambio profundo de actitud y el inicio de la construcción de un proceso, que permita bajar telones a lo anterior e iniciar de esta manera una nueva forma de vivir.

De esta manera contaríamos con un diagnóstico evaluativo, que debe ser dinámico, mediante el diseño de elementos de medición que nos vayan indicando desviaciones y la producción de correcciones, no se puede trabajar únicamente sobre la base de éxito o fracaso.

Es muy importante establecer vínculos con distintas entidades de la comunidad, a fin de construir canales de transferencia a los alumnos que han cumplido con la tarea/s de enseñanza y aprendizaje, a un nuevo contexto en el cual deben desenvolverse como resultado de haber cumplido con el tiempo de permanencia en el contexto de encierro.

Los aspectos que deben considerarse están vinculados a la actividad áulica, debiendo cumplimentarse los objetivos específicos del proyecto a fin de dar respuesta al objetivo general.

Lo desfavorable se dará si no se alcanzan los objetivos propuestos, y más aún si no hay una evaluación que nos vaya indicando los aciertos y errores, y la necesidad de capacidad de respuesta.

Algo importante a tener en cuenta es el desenvolvimiento del docente en espacio áulico del contexto de encierro, permanentemente debe revisar sus subjetividades, su punto de equilibrio, en función de que si me pongo a llorar con el que llora o le doy la espalda, no contribuyo al criterio de apoyo y colaboración que se debe mostrar permanentemente

La reflexión tiene como referencia situaciones concretas que se dan con motivo de la relación que se establece en el espacio de liber- 
tad el "aula" entre el profesor y los alumnos asistentes, con motivo de clases, o la presencia de un tribunal para tomar un examen final. La primera consideración que se debe tener es la necesidad de disponer de una mirada particular que permita diferenciar con claridad, una clase en la Facultad a una clase en el Servicio Penitenciario. La clase en el Servicio nos obliga a observar con rapidez cuál es el estado de ánimo de cada uno de los alumnos asistentes, todo esto en función de que el alumno asistente, vive impactado por distintas situaciones que ocurren en su lugar de alojamiento, como por ejemplo: en el lugar se alojan cincuenta detenidos y uno solo, el que asiste al "aula" es el que estudia, indudablemente si a ese alumno no se le permite disponer de un lugar diferenciado para estudiar, en su lugar de alojamiento, le es muy difícil hacerlo, lo cual indudablemente lo perjudica en su avance para la lectura y comprensión de los diferentes temas que el programa le exige que debe saber.

Si ese mismo alumno se inscribe para dar un examen final ante un tribunal, el tribunal debe disponer de una mirada particular que facilite el desarrollo de la exposición, considerando las diversas situaciones que ese alumno ha debido superar para llegar a esta instancia de un examen final. No disponer de esa mirada puede significar, frustración, deserción y otros resultados negativos con motivo de una falta de comprensión por parte de los profesores involucrados, del contexto en el cual se desenvuelve el alumno, la clase o el examen final.

\section{Referencias bibliográficas}

ACHILI, E. Investigar en Antropología Social. Los desafíos de transmitir un oficio. Rosario, Laborde Editor, 2005.

ARROYO, D. Politicas Sociales. Ideas para un debate necesario. Buenos Aires, Ediciones La Crujía, 2009.

CONSEJO FEDERAL de EDUCACIÓN. La Educación en Contextos de Privación de la Libertad en el Sistema Educativo Nacional. Documento Base, 2010. 
CORDERO MARTÍN, G; CORDERO RAMOS, N. y FERNÁNDEZ MARTÍN, M. (Comps). El Mosaico de la Intervención Social. Métodos y Conceptos en Trabajo Social. Colección Textos Universitarios, N 26. Sevilla, Aconcagua Libros, 2011.

DE ROBERTIS, C. Teoría del Trabajo Social (Grupos). España, Editorial Síntesis, 2006.

FERRANDO, J. Pensando en la Educación Popular. 3ª . Edición. Montevideo, Ed.

Nordan-Comunidad, 1991.

GALARZA, O. Misión de la Universidad y Profesionalización del Docente Universitario. UNCa, Facultad de Ciencias Agrarias. Maestría en Docencia Universitaria de Disciplinas Tecnológicas, 2008.

GARCÍA, M. La dimensión tecnológica. Significación y alcances en el nivel superior de enseñanza. UNCa, Facultad de Ciencias Agrarias, Septiembre de 2011.

GARCÉS, L. y LUCERO, M. (comps). Políticas Sociales -ciudadanía. Debates sobre una relación en tensión. $1^{\text {a }}$. Edición. San Juan, EFU, 2006.

KONOPKA, G. Social Group Work: a Helping Process Englewood Cliffs, N.J., Prentice Hall, 1963.

FREJTMAN, V. y HERRERA, P. Pensar la educación en contextos de encierro. Primeras aproximaciones a un campo en tensión, 2009.

OLIVA CARRERAS. M. A. La Dimensión Metodológica. Significación y Alcances en el Nivel Superior de Enseñanza. Trabajo Final. Maestría en Docencia Universitaria de Disciplinas Tecnológicas. 2010.

Recepción: 30/03/2014

Aceptación: 08/05/2014 